\title{
Effects of Sleep Deprivation on Pilot's Cognitive Behavior in Flight Simulation
}

\author{
Zhong-Qi Liu ${ }^{1}$, Qian-Xiang Zhou, ${ }^{1, *}$, and Fang Xie ${ }^{2}$ \\ ${ }^{1}$ School of Biological Science and Medical Engineering, \\ Beihang University, Beijing 100191, China \\ ${ }^{2}$ General Technology Department, \\ China North Vehicle Research Institute, Beijing 100072, China \\ liuzhongqi@buaa.edu.cn, zqxg@sjtu.edu.cn, \\ christie_xie@163.com
}

\begin{abstract}
This study examined the effects of $32 \mathrm{~h}$ of continuous $\operatorname{SD}$ (Sleep Deprivation) on cognitive behavior in simulated flight. Four subjects who were skilled in flight simulator took part in the experiment. Eye movement and flight parameters were measured in the following 5 time periods: 1100 on DAY 1, 1500,0400 on DAY 2, 1100, and 1500. Subject's cognitive workload and fatigue were assessed with method of NASA-TLX (national aeronautics and space administration-task load index) and RPE (rating of perceived exertion). Eye movement indices of average pupil area, average saccade amplitude and average saccade velocity decreased during the $32 \mathrm{~h}$ SD and they all showed significantly changes in the final SD while the index of average fixation time increased in the final SD. Flight performance that evaluated by four flight parameters of the deviation of height, pitch angle, yaw angle and tilt angle deteriorated during the $32 \mathrm{~h} \mathrm{SD}$, but not significantly. The feeling of fatigue and workload reported by subjects both increased during the $32 \mathrm{~h}$ SD. Effects of daily rhythm were also found, there were a obviously change at the hour of 0400. $32 \mathrm{~h}$ of SD has obvious effects on eye movement behaviors which have close relations to fatigue because of SD. The eye movement measurement can be served as a tool to continually monitor fatigue online.
\end{abstract}

Keywords: sleep deprivation, cognition, eye movement, flight performance.

\section{Introduction}

Flight is complex tasks that need high mental work. Adequate sleep is necessary for pilots' normal flights. Long-haul flights across time zones, night flights, night combat duty, repeated flights over the short term, irregular working hours will lead to pilots' SD. It is especially prevalent in the past air combat and will be more common in hightech war in the future.

$\mathrm{SD}$ is a major reason contributed to the flight accident and fatigue that played a role in over $12 \%$ of all US Air Force mishaps and is a recurring concern throughout

\footnotetext{
* Corresponding author. 
the aviation industry[1]. Insufficient sleep leads to a general slowing of response speed and increased variability in performance, particularly for simple measures of alertness, attention and vigilance[2-5], and aircrews are more likely to lower their standards of performance, suffer impairments in the ability to integrate information, and experience a reduced attention span that may lead to forgetting or ignoring important aspects of flying[6].There are mainly four kinds of methods of studying SD: subjective assessment method[7], physiological and biochemical measurements[8-10], performance measurement[11][12], psychology and behavioral method[13][14]. Subjective evaluation method of the data with a strong subjective component, and sometimes make people doubt its authenticity and reliability; physiological measurements and biochemical measurement usually need place device on the human body that make the subjects feel unwell and interfere the experimental task; in addition, various methods are generally measured in advance or post measurement and can't measure the actual flight task, so there was some deviation between the measurement data and the actual value.

The eyes are the windows of the soul; the information obtainment in the flight mission mainly depends on vision. In the SD process, the function of the visual system is bound to be affected. Researchers found that some eye movement parameters will change with fatigue[15]: blink rate generally increases with sleep deprivation and fatigue; pupil diameter typically decreases with sleep deprivation; saccadic velocity has been shown to decrease with sleep deprivation; mean saccade length increases with time-on-task; dwell time was shown to decrease with time-ontask.

Eye movement measures provide valuable information of not only fatigue and workload but also operators' attention, behavior performance and cognitive function that they all will be impaired during SD. The chief purpose of this study was to investigate changes in flight performance during extended wakefulness of over $30 \mathrm{hr}$ and to determine the relationship of eye movement behavior and fatigue and workload during SD.

\section{Method}

\subsection{Apparatus}

This study was conducted in the fixed base flight simulators which the prototype was a military aircraft of a high validity. IT was made up of the real cockpit, joystick, throttle lever, rudder, and true flight dynamics system and the simulator can complete the aircraft's acceleration, deceleration, pitch, yaw, tilt and all the basic fights. Flight performance measurements were collected in the flight simulator.

Eye movements were recorded by means of Eyelink II measuring system manufactured by Canada's SR Research CORP. Eyelink II system is a highbandwidth eye tracking system. Its spatial resolution is up to $0.01^{\circ}$ of view; its gaze error is less than $0.05^{\circ}$; the sampling frequency is $250 \mathrm{~Hz}$ with corneal reflection and pupil tracking mode. 


\section{$2.2 \quad$ Subjects}

Four young healthy male adults who were university students participated in this study (age range 18-22 years, mean age $=20$ ). They have been trained expertly for all kinds of basic flight tasks in above flight simulator. Screening excluded those having sleep difficulties who napped regularly in the daytime, and were on medication that affected sleep or sleepiness. Other requisites for inclusion were: no excessive daytime sleepiness; no smoking, drinking and gambling habits; no coffee and tea drinking habits; no neurological and psychiatric history; no other eye diseases. All had normal, or corrected normal vision. They had the procedures explained, signed consent forms, and were paid to participate.

\subsection{Task}

Subjects were asked to accomplish 1 minute level fight above the runway and with VFR (Visual Flight Rule) in a fine and no wind day. At the beginning of the fight, aircraft faced right the runway, the vertical height from the ground was $400 \mathrm{~m}$, and the level distance from the centre of the runway was $5400 \mathrm{~m}$ (Fig.1). The subject must keep the height of $400 \mathrm{~m}$ and the heading angle of $90^{\circ}$. Schedule of test flights for all four subjects is as follows: 1100 on Day 1, 1500, 0400 on Day 2, 1100 and 1500 so there were five test segments.

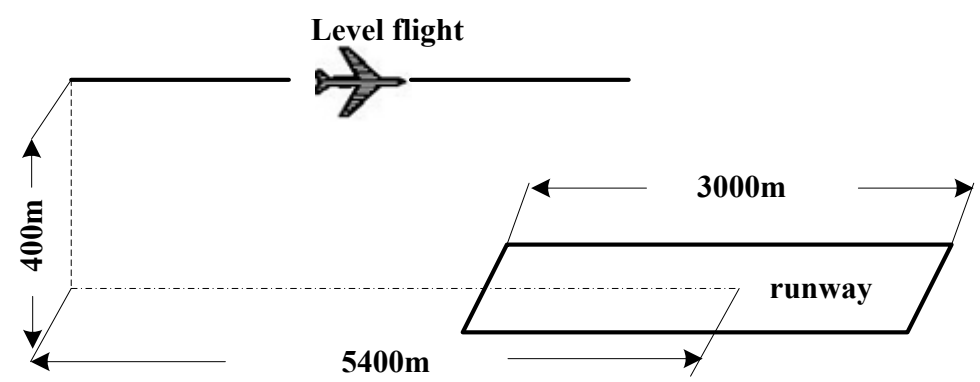

Fig. 1. Flight task schematic diagram

\subsection{Procedure}

Subjects were called to get up at 0700 and the SD began. Subjects were asked to keep wakeful after the entire test segment finished and it lasted almost $32 \mathrm{~h}$. After breakfast, they arrived at laboratory at about 0800. An informed consent agreement was signed by subjects and all upcoming experiment procedures were briefed to them.

After the calibrations of the eye movement measuring system, the subjects exercised the flight task several times to adapt the simulator. When the subjects said ok, the formal test began. Subjects were asked to complete four times fight on each segment. Flight parameters data for flight performance and eye movement data were recorded during the test. 
The feeling of drowsiness and fatigue were respectively measured with the SSS (Stanford Sleepiness Scale) and RPE (Rating of Perceived Exertion) scale before each formal test[16]. Cognitive workload was measured to investigate the subjects' state of workload with NASA-TLX (National Aeronautics and Space Administration-Task Load Index) scale after each formal test[5].

\section{$3 \quad$ Results}

In this study, the data of the time of 1100 on DAY 1 was the reference value and the other four segments' data were compared to it with paired t-test.

\subsection{Flight Performance}

Flight performance was evaluated by recording the four flight parameters which they were the deviation of height, pitch angle, yaw angle and tilt angle. The four parameters showed consistent change trend that they all got worse through the five $\mathrm{SD}$ segments, but the change didn't get significant level $(\mathrm{p}<0.05)$ except that the deviation of height(Fig.2). All the subjects' flight performance showed a similar pattern that the value of the flight parameters got worse at the time of 0400 than the former and latter of the performance. It may be the effect of the daily rhythm. It must be mentioned that it showed some individual difference of the performance between subjects. For instance: tilt angle tended to increase through 32hs SD and only the subject of NO 2 reached significant level $(\mathrm{p}<0.05)$ at 0400 while the others had not obvious change $(\mathrm{p}>0.05)$; At the final time, yaw angle of two subjects is obviously greater $t(p<0.05)$ than baseline values of 1100 on DAY 1 . It perhaps due to the difference of "SD resistance".

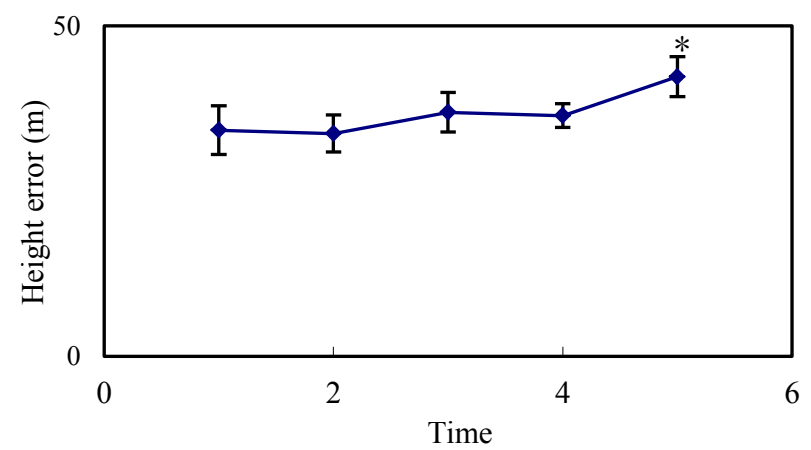

Note: * significantly different from the initial trial of 1100 on DAY $1(\mathrm{p}<.05)$.

$1-1100$ on DAY $1 ; 2-1500 ; 3-0400$ on DAY $2 ; 4-1100 ; 5-1500$

Fig. 2. Height deviation through $32 \mathrm{~h} \mathrm{SD}$ 


\subsection{SSS and RPE}

Subjective feeling of sleeping in SSS (Fig.3) score and fatigue in RPE (Fig.4) score were of similar trends which they were significantly increasing along with the SD time. SSS score and RPE score both reached a significant level $(\mathrm{p}<0.05)$ at 0400 . Seen form the figures of SSS and RPE, curves slope around the time of 0400 is obviously different. It may also the effect of daily rhythm.

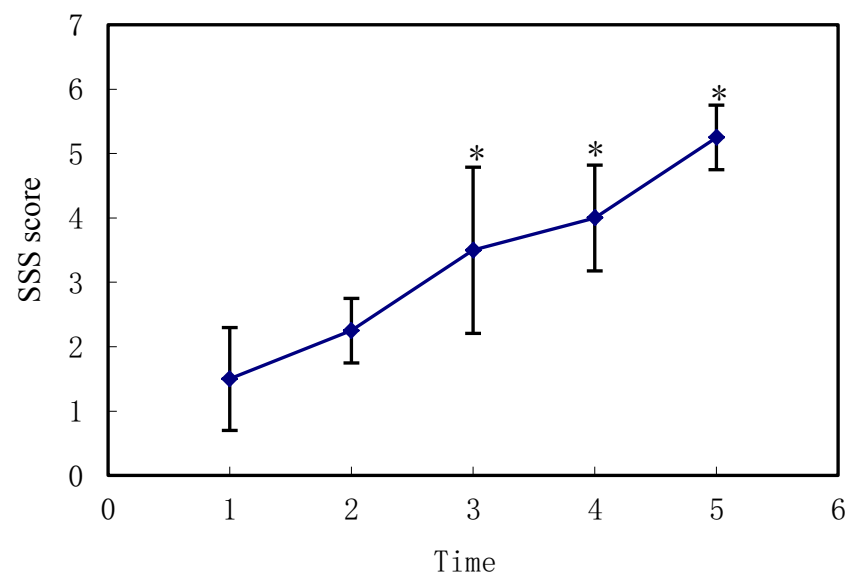

Note: * significantly different from the initial trial of 1100 on DAY $1(\mathrm{p}<.05)$. $1-1100$ on DAY $1 ; 2-1500 ; 3-0400$ on DAY $2 ; 4-1100 ; 5-1500$

Fig. 3. SSS change through $32 \mathrm{~h} \mathrm{SD}$

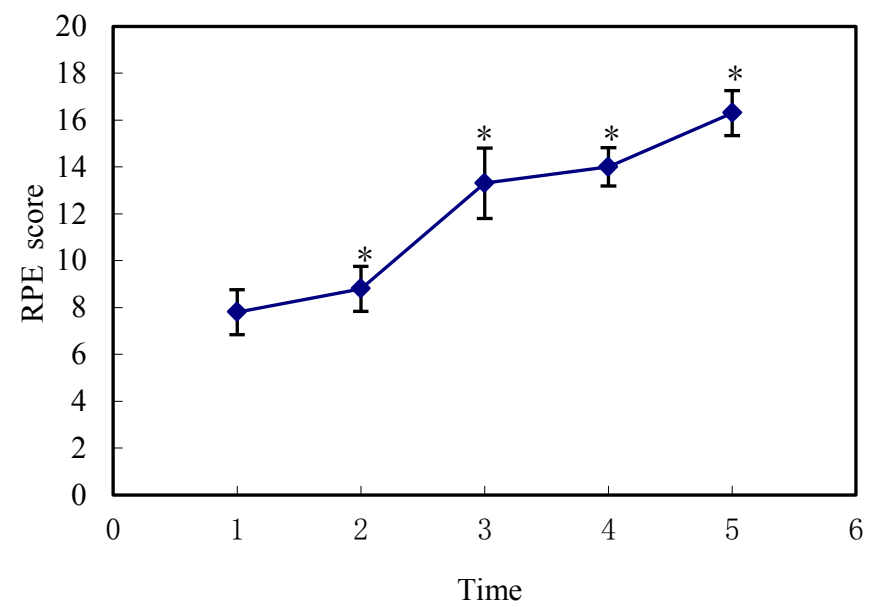

Note: * significantly different from the initial trial of 1100 on DAY $1(\mathrm{p}<.05)$.

$1-1100$ on DAY $1 ; 2-1500 ; 3-0400$ on DAY $2 ; 4-1100 ; 5-1500$

Fig. 4. RPE change through 32 hs SD 


\subsection{Cognitive Workload}

The workload of the subjects through the five test segments is assessed by the NASATLX score. The NASA-TLX was carried out based six parameters: mental demand (MD), physical demand (PD), temporal demand (TD), performance (Per), effort (E) and frustration level(FL). To clearly survey the change of the workload, six dimensions of the NASA-TLX were separately scored(Fig.5). They all increased with different degree through the whole SD, while MD changed most, followed by $\mathrm{E}$ and Per, FL changed least. Six dimensions all reached significant level $(\mathrm{p}<0.05)$ at the time of 0400 .

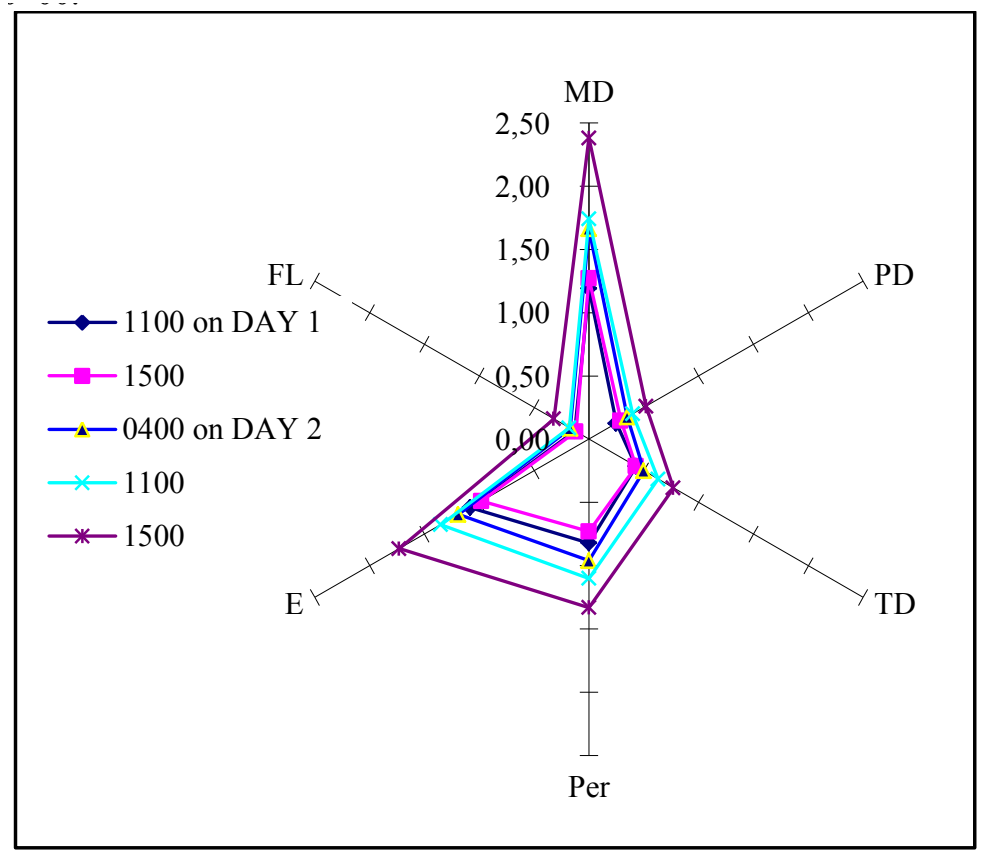

Fig. 5. NASA-TLX change through 32 hs SD

\subsection{Eye Movement Parameters}

As can be seen from Fig. 6, the pupil area of the four subjects showed a more consistent narrowing trend and their pupil change all reached significant level at different time that two subjects were of 0400 while one subject closed to the edge significant level at 1100 on DAY 2 and then reached significant level $(\mathrm{p}<0.05)$ at final time. Only one subject was some of different from the others, his pupil area increased at 1500 on DAY 1am and then decreased the following time and reached significant level.

Average saccade amplitude of four subjects decreased during $32 \mathrm{~h} \mathrm{SD}$ and reached significant level $(\mathrm{p}<0.05)$ in different time that two subjects were at the final time of SD and the other two were at 1100 on DAY 2. 
Four subjects show a consistent decreasing trend of the index of the average saccade velocity. Three subjects reached significant level $(\mathrm{p}<0.05)$ at 11:00 on DAY 2 while one subject was at the final time of SD.

The indicator of average fixation time increased through the $32 \mathrm{~h} \mathrm{SD}$. Four subjects all reached significant level at the final time of SD.

Indicators of fixation frequency, peak saccade velocity and vengeance did not make any obvious and regular changes in this study.

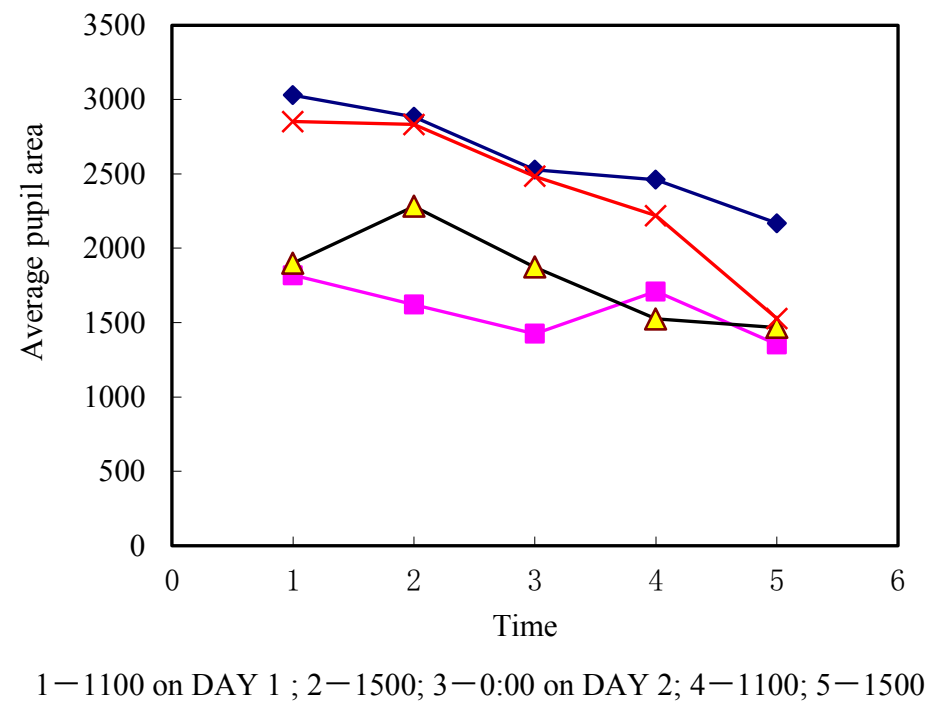

Fig. 6. Pupil change through 32 hs SD

\section{Discussion}

The direct effect of SD was enhanced fatigue and increased sleepiness. The correlation analysis between RPE and SSS showed a high degree of correlation $(\mathrm{r}=$ 0.987). They changed eye movement behavior and result in the pupil's miosis, decreasing saccade amplitude and saccade velocity while the average fixation time became longer. These changes might have two reasons. First, Sustained wakefulness leads to the physical fatigue of eye muscle. Eye movement load is a function of time, the longer of the watching time the greater accumulation of visual load and so as to the accumulation and generation of eye muscle fatigue. Second, sustained wakefulness leads to mental fatigue. Brain made various basic mental activities in waking state which they include exchanges with other persons, individual psychological activity, carrying out the experimental tasks and other activities. With the continued awake time, mental fatigue gradually accumulated and resulted in the alteration of person's eye movement behavior. 
Fatigue is an important factor affecting pupil size. Lowenstein pointed out that pupil diameter was the largest after a good rest and narrowed with the people's fatigue[17]. Russo studied the effects of SD on human eye movement behavior and founded that the average saccade velocity decreased significantly [18]. He pointed out that saccade velocity index can be a good indicator for the alert degree in operating environment and lowered saccade velocity means that the operator was in fewer alerts that increased the probability of the accident. Gennaro studied the scan behavior during 40 hours SD. The results showed that the scan velocity and saccade amplitude reduced [19]. It can be seen that the results of this study is consistent with that of some researchers.

Average fixation time did not change in Schleicher study [20]. It might be relate to the monotonous and dull tasks because the fixation point of $150 \mathrm{~ms}$ and $900 \mathrm{~ms}$ increased and the fixation point less than $150 \mathrm{~ms}$ didn't relate to cognitive processing while the fixation point more than $900 \mathrm{~ms}$ were relation to the gradually decreasing interesting due to the monotony tasks, so the average fixation time almost had no change. In this study, flight simulation operation was a complex task. Subjects have been trying to operate, but because of the accumulating fatigue, their ability of information processing speed gradually slowed down led to the longer dwell time. NASA-TLX score of mental demand also showed that flight simulation has a higher demand to the people's mental activity.

In this study, flight performance showed decreasing trend, but it didn't reach significant level $(\mathrm{p}<0.05)$ which may be relate to the not long enough time of SD. Some eye movement indicators have shown a significant change which illuminate that eye movement parameters were more sensitive to SD than flight performance. This may be more meaningful for fatigue monitoring and early warning because of SD. In real flight, pilots can be continuously measured in real time with modern advanced eye movements tracking technology. An early warning can be made for the pilot's fatigue or sleepiness and ensure safety driving.

In this study, almost all the experiment results were influenced by people's circadian rhythms which it have been founded in others studies[1][15]. It suggests that people who need night operations, such as various drivers and various monitoring staff, must take some action to keep alert at Time of 0400. To do that, one can make themselves have a rest or nap in the afternoon or ask the operations staff to remind themselves or remind them by communication in their physical low point.

\section{Conclusion}

In conclusion, the results of this study provide an important glimpse of pilot's cognitive behavior during about $32 \mathrm{hr}$ of extended wakefulness. It can be seen that subjects' flight performance and eye movement performance were affected by the increasing of the subjects' fatigue and cognitive workload. Eye movement behavior is more easily suffered than that of flight performance during SD. The characteristic of the sensitiveness of the eye movement behavior to SD can be utilized to monitor pilot in real time who are operating which can forecast and warn early of pilot's fatigue and 
sleepiness. With that characteristic, flight performance predication and evaluation can also be made and it is the issue of further study.

Acknowledgement. This work is supported by the Technology Foundation of National Science (A0920132003) and the Natural Science Foundation of China (31170895).

\section{References}

1. Lopez, N., Previc, F.H., Fischer, G., et al.: Effects of sleep deprivation on cognitive performance by United States Air Force pilots. Journal of Applied Research in Memory and Cognition 1, 27-73 (2012)

2. Killgore, W.D.: Effects of sleep deprivation on cognition. Progress in Brain Research 185, $105-129$ (2010)

3. Whitney, P., Hinson, J.M.: Measurement of cognition in studies of sleep deprivation. Progress in Brain Research 185, 37-48 (2010)

4. Kim, H.J., Kim, H., Park, K.D., Choi, K.G., Lee, H.W.: A survey of sleep deprivation patterns and their effects on cognitive functions of residents and interns in Korea. Sleep Medicine 12, 390-396 (2011)

5. Tomasko, J.M., Pauli, E.M., Kunselman, A.R., Haluck, R.S.: Sleep deprivation increases cognitive workload during simulated surgical tasks. The American Journal of Surgery 203, 37-43 (2012)

6. Perry, I.C.: Helicopter aircrew fatigue. AGARD Advisory Report No. 69

7. Cao, X.L.: Experimental Study of the Attention Character and Subjective Assessment Methods on Mental Fatigue. PhD thesis of the Fourth Military University (2003) (in Chinese)

8. Belyavin, A., Wright, N.A.: Changes in electrical activity of the brain with vigilance. Electroencephalograph. Clin. Neurophysiology 66(2), 137-144 (1987)

9. Fernstrom, J.D., Fernstrom, M.H.: Exercise, serum free tryptophan, and central fatigue. The Journal of Nutrition 136(2), 553-559 (2006)

10. Ohta, M., Hirai, N., Ono, Y., et al.: Clinical biochemical evaluation of central fatigue with 24-hour continuous exercise. The Japanese Journal of Clinical Pathology 53(9), 802-809 (2005)

11. Zhang, Q., Cheng, J.N., Yang, P.J., et al.: Preliminary Study on Balance Function Quantitative Assessment in Human. Chinese Journal of Rehabilitation 13(2), 49-52 (1998) (in Chinese)

12. Wang, S.Y., Zhang, L.F., Cheng, J.H., Wang, X.B.: Time-frequency analysis of heart rate variability during head up tilt. Journal of the Fourth Military Medical University 22(4), 301-305 (2001) (in Chinese)

13. Ji, H.G., Zhang, L., Wang, H.M.: Functional change after 24 hour sleep deprivation. Chinese Journal of Behavioral Medical Science 7(4), 258-259 (1998)

14. Yue, P., Miao, D.M., Huang, P.E., et al.: Influence of 32-hour sleep deprivation on selective attention. Journal of Fourth Military Medical University 25(4), 378-381 (2004)

15. Previc, F.H., Lopez, N., Ercoline, W.R., et al.: The Effects of Sleep Deprivation on Flight Performance, Instrument Scanning, and Physiological Arousal in Pilots. The International Journal of Aviation Psychology 19(4), 326-346 (2009) 
16. Bailes, S., Libman, E., Baltzan, M., et al.: Brief and distinct empirical sleepiness and fatigue scales. Journal of Psychosomatic Research 60, 605-613 (2006)

17. Lowenstein, O., Locwenficld, I.E.: The sleep-waking cycle and pupillary activity. Animals of the New York Academy of Sciences 117, 142-156 (1964)

18. Russo, M., Thomas, M., Sing, H., et al.: Saccadic velocity and pupil constriction latency changes in partial sleep deprivation, and correlations with simulated motor vehicle crashes. Sleep 22(1), 297-298 (1999)

19. Gennaro, L.D., Ferrara, M., Urbani, L., et al.: Oculomotor impairment after 1 night of total sleep deprivation: a dissociation between measures of speed and accuracy. Clinical Neurophysiology 111(10), 1771-1778 (2000)

20. Schleicher, R., Galley, N.: Blinks and saccades as indicators of fatigue in sleepiness warners: looking tired? Ergonomics 51(7), 982-1010 (2008) 